\title{
Non-geometric heterotic backgrounds and 6D SCFTs/LSTs
}

\author{
Anamaría Font \\ Facultad de Ciencias, Universidad Central de Venezuela, A.P.20513, Caracas 1020-A, Venezuela \\ E-mail: afontefisica.ciens.ucv.ve
}

\section{Iñaki García-Etxebarria}

Max-Planck-Institut für Physik, Föhringer Ring 6, 80805 München, Germany

E-mail: inaki@mpp.mpg.de

\section{Dieter Lüst}

Max-Planck-Institut für Physik, Föhringer Ring 6, 80805 München, Germany; and ASC for Theoretical Physics, Theresienstraße 37, 80333 München, Germany

E-mail: dieter.luestalmu.de

\section{Stefano Massai}

Enrico Fermi Institute, University of Chicago, 5640 S Ellis Ave, Chicago, IL 60637, USA

E-mail: massai@uchicago.edu

\section{Christoph Mayrhofer*}

ASC for Theoretical Physics, Theresienstraße 37, 80333 München, Germany

E-mail: christoph.mayrhofer@lmu.de

\begin{abstract}
We study $\mathscr{N}=(1,0)$ six-dimensional theories living on defects of non-geometric backgrounds of the $E_{8} \times E_{8}$ and the $\operatorname{Spin}(32) / \mathbb{Z}_{2}$ heterotic strings. Such configurations can be analyzed by dualizing to F-theory on elliptic K3-fibered non-compact Calabi-Yau threefolds. The majority of the resulting dual threefolds turn out to contain singularities which do not admit a crepant resolution. When the singularities can be resolved crepantly, the theories living on the defect are explicitly determined and reveal a form of duality in which distinct defects are described by the same IR fixed point. In particular, a subclass of non-geometric defects corresponds to SCFTs/LSTs arising from small heterotic instantons on ADE singularities.
\end{abstract}

Corfu Summer Institute 2016 "School and Workshops on Elementary Particle Physics and Gravity" 31 August - 23 September, 2016

Corfu, Greece

\footnotetext{
* Speaker.
} 


\section{Introduction}

Since the early studies of string compactifications most work has been done in the supergravity regime. However it is well known that string vacua can be much richer. Our motivation in this article is to take a step away from vacua where the background can be understood geometrically by considering a classical supergravity compactification. The aim is both to learn more about the non-classical and non-geometrical properties of string theory, and to gain some insight about the broader set of allowed string vacua.

In this paper we consider a class of heterotic string vacua which are very non-classical, involving compactifications on "spaces" that cannot be globally described as geometries, while remaining accessible thanks to duality with F-theory [1]. In this way we can probe many of the properties of the heterotic string away from the classical regime where it is usually studied. More concretely, we will focus on cases where the compactification space for the heterotic string at a generic point is locally geometric, and described by a $T^{2}$ fibration. The non-classical nature of the background arises from the patching between local descriptions, which entails non-trivial elements of the T-duality group acting on the $T^{2}$. Such fibrations will in general have defects where a local description in terms of the heterotic string on a smooth background is no longer possible.

For concreteness, we consider the compactification of the heterotic string to six dimensions so that we have locally a $T^{2}$ fibration over a complex one-dimensional base. At certain points of the base there are defects and our goal is to describe the low-energy dynamics living on the defects themselves. This is achieved by dualizing the configuration to F-theory, where the dynamics on the defects can be characterized by purely geometric means. Generically, the F-theory background dual to a given defect on the heterotic side are highly singular. In some cases we are able to resolve the singularity crepantly by performing a finite number of blow-ups in the base of the fibration. For all the cases where this resolution is possible we construct the resulting smooth geometry. The blow-ups correspond to giving vevs to tensor multiplets of the $6 \mathrm{~d}(1,0)$ theory on the defect, such that it flows to a Lagrangian description in the IR. For the cases that can be resolved the emerging theories can be related to $6 \mathrm{~d}$ SCFTs, such as the long known theories of small instantons on an ADE singularity [2, 3], or 6d SCFTs that have been recently classified $[4,5,6]$. Actually, the resulting theories fall into configurations whose UV completions are conjectured to be $6 \mathrm{~d}$ little string theories (LSTs) [7, 8, 9] since they have distinctive properties of LSTs [10] such as a mass scale and T-duality upon circle compactification. Moreover, there is a prescription to extract $6 \mathrm{~d}$ SCFTs embedded in the LSTs [9].

The paper is organized as follows: In section 2, after recalling the basics of heterotic compactifications on $T^{2}$, we review the formulation of heterotic/F-theory duality in terms of a map between genus-two curves and K3 surfaces. Moreover, we discuss how it can be used to study nongeometric heterotic backgrounds in terms of K3 fibered Calabi-Yau three-folds. In section 3, we first explain the procedure to resolve singularities and then apply the formalism to local heterotic degenerations which admit a geometric description in some duality frame. We also discuss truly non-geometric models and describe a kind of duality between different non-geometric and geometric defects. In section 4 we summarize the classification of all possible local heterotic models, both geometric and non-geometric, admitting F-theory duals that can be resolved crepantly into smooth Calabi-Yau three-folds. We end with some final comments. 


\section{Non-geometric heterotic vacua and F-theory}

In this section we describe what we actually mean by non-geometric heterotic string vacua. We construct them in two steps: first we compactify the ten-dimensional string theory on a two-torus; then we use the duality group of the moduli to get a non-trivial, i.e. non-geometric, identification of these fields when going along a loop of non-trivial homotopy. We will also review the duality between the heterotic string and F-theory $[11,12,13]$, in preparation for section 3 where we use the F-theory representation to get a better handle on the low-energy degrees of freedom of the non-geometric heterotic vacua.

\subsection{Heterotic string on $T^{2}$}

From the compactification of the heterotic string on a torus $T^{2}$, we obtain the following moduli fields in eight dimensions:

- A complexified Kähler modulus $\rho=\int_{T^{2}} B+\omega \wedge \bar{\omega}$ with $B$ the Kalb-Ramond two-form and $\omega$ the holomorphic one-form of the torus which can be obtained from the metric on $T^{2}$.

- The complex structure modulus $\tau=\int_{b} \omega / \int_{a} \omega$, where $a$ and $b$ denote the two generators of the non-trivial one-cycles of the torus.

- Furthermore, there are 16 complex Wilson line moduli from the Cartan generators of the non-abelian gauge group of the heterotic string, i.e. $\beta^{i}=\int_{a} A^{i}+i \int_{b} A^{i}$.

As it is well known there are dualities among torus compactifications. Therefore, the local moduli space $O(2 ; \mathbb{R}) \times O\left(2+n_{W L} ; \mathbb{R}\right) \backslash O\left(2,2+n_{W L} ; \mathbb{R}\right)$ of the heterotic $T^{2}$ compactification becomes the Narain space [14]

$$
O(2 ; \mathbb{R}) \times O\left(2+n_{W L} ; \mathbb{R}\right) \backslash O\left(2,2+n_{W L} ; \mathbb{R}\right) / O\left(2,2+n_{W L} ; \mathbb{Z}\right),
$$

with $n_{W L}$ the number of Wilson line moduli switched on. The cases of interest to us are those with none or one non-vanishing Wilson line modulus. In these situations the heterotic/F-theory duality map is known explicitly and can be used to analyse the heterotic vacua.

\subsection{Vacua with varying moduli fields}

To set the ground for the second step of our compactification we rewrite the above moduli space. For $n_{W L}=1$ we can map ${ }^{1}$ the Narain moduli space to the Siegel upper half plane of genustwo curves

$$
\mathbb{H}_{2}=\left\{\Omega=\left(\begin{array}{cc}
\tau & \beta \\
\beta & \rho
\end{array}\right) \mid \mathfrak{I}(\operatorname{det}(\Omega))>0 \wedge \mathfrak{I}(\rho)>0\right\}
$$

quotiented by an $S p(4, \mathbb{Z})$-action

$$
\Omega \rightarrow(A \Omega+B)(C \Omega+D)^{-1} \quad \text { with } \quad\left(\begin{array}{ll}
A & B \\
C & D
\end{array}\right) \in S p(4, \mathbb{Z}) .
$$

\footnotetext{
${ }^{1}$ We should note that this map is a priori only well-defined from $\mathrm{H}_{2} / S p(4, \mathbb{Z})$ to the Narain moduli space. Only on $O(2 ; \mathbb{R}) \times O(3 ; \mathbb{R}) \backslash O(2,3 ; \mathbb{R}) / S O^{+}(2,3 ; \mathbb{Z})$, where $S O^{+}(2,3 ; \mathbb{Z})$ is an order four subgroup of $O(2,3 ; \mathbb{Z})$, it becomes a well-defined bijective map.
} 
The advantage of this rewriting is firstly that the above moduli $\rho, \tau$, and $\beta$ are just the entries of $\Omega$ as denoted in (2.2). Secondly, in this representation it is natural to assign to every moduli space point $p$ a genus-two curve $\mathscr{C}_{p}$ with complex structure

$$
\Omega_{i j}=\int_{b_{i}} \omega_{j}
$$

Here $a_{i}, b_{i}$, and $\omega_{j}$ are respectively the non-trivial one-cycles and holomorphic one-forms of $\mathscr{C}_{p}$, with normalization $\int_{a_{i}} \omega_{j}=\delta_{i j}$. In this way we obtain a geometrification of our moduli.

In the vein of F-theory, we use this geometrification to construct six-dimensional heterotic string vacua with varying moduli fields. Therefore, we let the heterotic torus fibration vary adiabatically along two real dimensions or one complex dimension which we parametrize by $t$. For the moduli to fulfill the (BPS) equations of motion they must vary holomorphically in $t$. To obtain the wanted (globally) non-geometric configurations, we puncture the $t$-plane and allow for ' $\operatorname{Sp}(4, \mathbb{Z})$ patchings' of the moduli fields when encircling the punctures, i.e. we identify dual theories when going along a non-contractible loop. ${ }^{2}$ Since every $\operatorname{Sp}(4, \mathbb{Z})$-orbit in $\mathrm{H}_{2}$ is identified with exactly one genus-two curve, holomorphic genus-two fibrations are the natural candidates to encode such vacua. If such a fibration is non-trivial it will degenerate in complex codimension one. These degeneration points are the punctures of the $t$-plane and the kind of singularity is in one-to-one relation with a certain $S p(4, \mathbb{Z})$-duality transformation on the moduli.

Now, it is a happy coincidence that all the degenerations of genus-two curves were classified by Ogg-Namikawa-Ueno $[15,16]$. This mathematical result gives us a huge list of non-geometric heterotic string vacua. However, to understand them we have to make sense of the singularity loci of the fibration. In string theory we are used to the appearance of new light degrees of freedom which cure the theory when we run towards a seeming singularity. Since, we do not know how to do such an analysis for these specific compactifications directly on the heterotic side, we use the duality with F-theory to study them. The localised physical objects, which lie at the center of the genus two-degeneration, are called $T$-fects, which is short for $T$-duality defect.

\subsection{Mapping the setting to F-theory}

Since the invention of F-theory [1] it is known that the heterotic string on $T^{2}$ is dual to F-theory compactified on $\mathrm{K} 3$ [11]. This duality is best understood in the large volume/stable degeneration limit $[17,11]$. This special point in moduli spaces means that on the heterotic side we have $\rho \rightarrow i \infty$ and on the F-theory side the K3 degenerates into two 'del Pezzo nine' surfaces which intersect each other along a $T^{2}$. The identification of the moduli data is now as follows: $\tau$ is the complex structure of the F-theory $T^{2}$ at the intersection, and the Wilson lines are encoded in the intersection points (spectral cover data [18]) of the respective nine exceptional curves of the two $\mathrm{dP}_{9}$ 's with the $T^{2}$. Unfortunately such a detailed identification with all Wilson line moduli non-vanishing exists only for this special point in moduli space. However, for the case which we consider in this article, i.e. only one Wilson line non-vanishing, we can even do better. In this case the map between the moduli fields on both sides is known along the whole moduli space [19, 12].

\footnotetext{
${ }^{2}$ Non-geometric configurations usually refer to situations where the metric is identified with its inverse along a non-trivial path. However, in our case generically we have mixings of all three moduli of which $\rho \rightarrow 1 / \rho$ is just a subgroup.
} 
For $n_{W L}=1$, in the $E_{8} \times E_{8}$ heterotic string, the hypersurface describing the elliptically fibered F-theory $\mathrm{K} 3$ takes the following form:

$$
y^{2}=x^{3}+\left(a u^{4} v^{4}+c u^{3} v^{5}\right) x z^{4}+\left(b u^{6} v^{6}+d u^{5} v^{7}+u^{7} v^{5}\right) z^{6}
$$

where $x, y, z$ and $u, v$ are the homogeneous coordinates of fiber ambient variety $\mathbb{P}_{2,3,1}$ and the base $\mathbb{P}^{1}$, respectively. This $\mathrm{K} 3$ has a $I I^{*}$ singularity at $v=0$ and a $I I I^{*}$ at $u=0$ which correspond to an $E_{8}$ and an $E_{7}$ gauge group, respectively-matching the remaining unbroken heterotic gauge group after switching on one Wilson line modulus. Furthermore, the Picard number of this manifold is 17. Therefore, its complex structure moduli space [20] exactly agrees with the heterotic moduli space. ${ }^{3}$ As mentioned already, the map between the complex structures of the F-theory K3 (2.5) and the heterotic moduli (2.2) is explicitly known and given by [19, 12]:

$$
a=-\frac{1}{48} \psi_{4}(\Omega), \quad b=-\frac{1}{864} \psi_{6}(\Omega), \quad c=-4 \chi_{10}(\Omega), \quad d=\chi_{12}(\Omega) .
$$

with $\psi_{4}, \psi_{6}, \chi_{10}$, and $\chi_{12}$ the genus-two Siegel modular forms [21] of weight four, six, ten, and twelve, respectively. The modularity of the forms is meant with respect to the $S p(4, \mathbb{Z})$ transformation (2.3).

As we pointed out already, we are interested in an understanding of the physics of the vacua given by the list of genus-two degenerations. In their classification Namikawa and Ueno [16] give the genus-two singularities explicitly in terms of fibrations of hyperelliptic curves, i.e. sextic equations of the form

$$
y^{2}=c_{6}(t) x^{6}+c_{5}(t) x^{5}+\ldots+c_{1}(t) x+c_{0}
$$

with the $c_{i}(t)$ 's functions (or sections) of $t$. Furthermore, all the hyperelliptic curve fibrations are in a canonical form, in the sense that the singularity lies at $t=0$. Having the genus-two fibrations in the form of (2.7) is very convenient for us because we can use the relations between the genus-two Siegel modular forms and the Igusa-Clebsch invariants ${ }^{4} I_{2}, I_{4}, I_{6}, I_{10}$ of the sextic [23],

$$
\begin{array}{lr}
I_{2}\left(c_{i}\right)=\frac{\chi_{12}(\Omega)}{\chi_{10}(\Omega)}, & I_{4}\left(c_{i}\right)=2^{-4} \cdot 3^{-2} \psi_{4}(\Omega), \\
I_{6}\left(c_{i}\right)=2^{-6} \cdot 3^{-4} \psi_{6}(\Omega)+2^{-4} \cdot 3^{-3} \frac{\psi_{4}(\Omega) \chi_{12}(\Omega)}{\chi_{10}(\Omega)}, & I_{10}\left(c_{i}\right)=2^{-1} \cdot 3^{-5} \chi_{10}(\Omega),
\end{array}
$$

to write down the $\mathrm{K} 3$ coefficients $a, b, c, d$ as functions of the sextic coefficients $c_{i}$. In the end, we obtain for every genus-two singularity a K3 fibration over the $t$-plane with the K3 fibre degenerating at $t=0$. In the next section we will look at these F-theory singularities and try to resolve them if possible. In this way we get some insight about the objects which live at these six-dimensional loci.

The F-theory K3 dual to the $\operatorname{Spin}(32) / \mathbb{Z}_{2}$ heterotic string compactified on $T^{2}$ with one Wilson line is also known [24, 12]. It is described by:

$$
y^{2}=x^{3}+v\left(u^{3}+a u v^{2}+b v^{3}\right) x^{2} z^{2}+v^{7}(c u+d v) x z^{4} .
$$

\footnotetext{
${ }^{3}$ Note that this is obviously also true for the cases with $n_{W L}>1$ and one of the reasons why these two theories are dual to each other.

${ }^{4}$ See appendix C of [22] for the explicit form of the Igusa-Clebsch invariants in terms of the coefficients of the sextic.
} 
Putting the equation into Weierstraß form and computing the discriminant shows that this $\mathrm{K} 3$ has singularities of type $\mathrm{I}_{10}^{*}(S O(28))$ at $v=0$, and of type $\mathrm{I}_{2}(S U(2))$ at $c u+d v=0$, for generic coefficients. Hence, the gauge group is $\operatorname{Spin}(28) \times S U(2) / \mathbb{Z}_{2}$. When $c \equiv 0$ the group enhances to $\operatorname{Spin}(32) / \mathbb{Z}_{2}$.

Before we finish this section and go on to the resolutions, we should note that the map from the F-theory side to the heterotic side is much more involved, see for instance [25] for a first step into this direction.

\section{Resolution of singularities: procedure and examples}

Having established the duality map between the heterotic vacua and the F-theory vacua, we look now at the resolution of the singularities. Put differently in terms of F-theory language, we move onto the tensor branch of the theory to get an insight into the degrees of freedom which lie at the heart of the genus-two degenerations.

\subsection{General strategy}

We will always work with a Weierstraß model in the following, i.e. the elliptic fibration is always represented in terms of a hypersurface equation of the form:

$$
y^{2}=x^{3}+f\left(\xi_{i}\right) x z^{4}+g\left(\xi_{i}\right) z^{6}
$$

where $x, y, z$ are again the homogeneous coordinates of $\mathbb{P}_{2,3,1}$ and $f$ and $g$ are sections of some line bundles over the base $B \ni \xi_{i}$. For the elliptic fibration to be Calabi-Yau the line bundles of $f$ and $g$ have to be $K_{B}^{-4}$ and $K_{B}^{-6}$, respectively, with $K_{B}$ the canonical bundle of the base.

Throughout this article, we will call a singularity resolved if the elliptic curve has only minimal singularities [26] (or Kodaira type singularities) along the base, i.e. there are no points along the discriminant locus of (3.1) where $f$ vanishes to order four or higher and simultaneously $g$ to order six or higher. Furthermore, in our examples the base on the F-theory side is given by a (trivial) $\mathbb{P}^{1}$ fibration over the $t$-plane. Since the coefficient in front of the $u^{7} v^{5}$ term in (2.5) is constant, there is no such non-minimal singularity along $v=0$. Therefore, we only have to look at the $u$ - $t$-patch for such points and, as it turns out, in the beginning there is just one non-minimal singularity namely at $u=t=0$. To get rid of this non-minimal point we follow [2] and blow-up the base at this point. However, we do this in a rather toric manner by introducing the maximal amount of crepant ${ }^{5}$ blowups at once at the non-minimal point and not in a blow-up after blow-up procedure. Afterwards we search for non-minimal points along the newly introduced exceptional curves and, if necessary, apply our 'toric blow-up procedure' again. The analysis can also be applied to the dual F-theory $\mathrm{K} 3$ of the $\operatorname{Spin}(32) / \mathbb{Z}_{2}$ heterotic string, described by the equation (2.9).

\footnotetext{
${ }^{5}$ Crepant in the sense that the proper transform of the hypersurface equation (2.5) after the base blow-up is still Calabi-Yau. We do not claim that the canonical class of the base does not change which is obviously wrong.
} 


\section{Toric blow-up procedure}

As a first step, we choose local affine coordinates $\xi_{i}$ on $B$ such that the non-minimal singularity lies at $\xi_{1}=\xi_{2}=0$. We expand the sections $f$ and $g$ in these coordinates,

$$
f=\sum_{i} f_{i} \xi_{1}^{m_{i}^{1}} \xi_{2}^{m_{i}^{2}}, \quad g=\sum_{i} g_{i} \xi_{1}^{l_{i}^{1}} \xi_{2}^{l_{i}^{2}}
$$

and collect the minimal exponents $\mathbf{m}_{i}$ and $\mathbf{l}_{i}$. Next we look for all 'toric' blow-up [27] directions $\mathbf{n}_{j}$ which are crepant. For the elliptic fibration to remain Calabi-Yau, the blow-up $\mathbf{n}$ must involve the fibre coordinates $x$ and $y$ too:

$$
\xi_{1}, \xi_{2}, x, y \quad \mapsto \quad e^{n_{1}} \tilde{\xi}_{1}, e^{n_{2}} \tilde{\xi}_{2}, e^{2\left(n_{1}+n_{2}-1\right)} \tilde{x}, e^{3\left(n_{1}+n_{2}-1\right)} \tilde{y} .
$$

Hence, the canonical class of the ambient variety after the blow-up is given by $E$ times the last column in the following table:

$$
\begin{array}{|c|c|c|c|c|c|c|}
\hline & \xi_{1} & \xi_{2} & x & y & e & \sum \\
\hline E & n_{1} & n_{2} & 2\left(n_{1}+n_{2}-1\right) & 3\left(n_{1}+n_{2}-1\right) & -1 & 6\left(n_{1}+n_{2}-1\right) \\
\hline
\end{array}
$$

where $-E$ is the divisor class of the exceptional divisor $e=0$. Since we demand that our resolution of the Weierstraß equation is crepant, $e^{6\left(n_{1}+n_{2}-1\right)}$ must factor off the hypersurface equation (3.1) when we take its proper transform after applying (3.3). This amounts then to the constrains

$$
\left(m_{i}^{1}-4\right) n_{1}+\left(m_{i}^{2}-4\right) n_{2}=: \tilde{\mathbf{m}}_{i} \cdot \mathbf{n} \geq-4 \quad \text { and } \quad\left(l_{i}^{1}-6\right) n_{1}+\left(l_{i}^{2}-6\right) n_{2}=: \tilde{\mathbf{l}}_{i} \cdot \mathbf{n} \geq-6
$$

which must be fulfilled for all $\tilde{\mathbf{m}}_{i}$ and $\tilde{\mathbf{I}}_{i}$. The solutions to these inequalities is the set of toric blow-ups that we introduce.

After this resolution step, we have to check whether there are no non-minimal points along the just introduced exceptional curves. If there are any of them, we have to repeat the procedure at these points. We are done if we got rid of all the non-Kodaira type singularities.

\subsection{Geometric models: small instantons on ADE singularities}

In this section, we consider resolutions of heterotic models which on the genus-two side have a Namikawa-Ueno (NU) degeneration $\left[\mathrm{I}_{n-p-0}\right],\left[\mathrm{I}_{n}-\mathrm{I}_{p}^{*}\right]$ and $\left[\mathrm{K}-\mathrm{I}_{n}\right]$, with $\mathrm{K}=\mathrm{II}^{*}, \mathrm{III}^{*}, \mathrm{IV}^{*}[16]$. Here we use the notation $\left[\mathrm{K}_{1}-\mathrm{K}_{2}-0\right] \equiv\left[\mathrm{K}_{1}-\mathrm{K}_{2}\right]$ for the Namikawa-Ueno degenerations. Based on the monodromy action on the moduli and the Bianchi identity, these models are expected to describe heterotic compactifications with small instantons sitting at ADE singularities. For example, in the $\left[\mathrm{II}^{*}-\mathrm{I}_{n}\right]$ model the monodromy is

$$
\tau \rightarrow-\frac{1}{1+\tau}, \quad \rho \rightarrow \rho+n-\frac{\beta^{2}}{1+\tau}, \quad \beta \rightarrow \frac{\beta}{1+\tau} .
$$

When the Wilson line value $\beta$ is turned off, this is precisely the monodromy of a II* type fiber of the $\tau$ fibration. In general, in $\left[\mathrm{K}-\mathrm{I}_{n}\right]$ models it follows that there is a number $k=\mu(c)$ of small instantons on top of the K-type singularity.

The starting point is the genus-two model given in the NU classification. The next step is to compute the Igusa-Clebsch invariants that determine the $a, b, c$, and $d$ coefficients entering in the 
dual $\mathrm{K} 3$ on the F-theory side. In table 1 we collect the defining equations of the ADE NU models together with the vanishing degrees of the coefficients $a, b, c$, and $d$. On the F-theory side there are points where the vanishing orders of $f, g$, and $\Delta$ in the Weierstraß model are non-minimal, so we proceed to resolve as explained in the preceding section.

\begin{tabular}{|c|c|c|c|c|c|c|}
\hline sing. & NU type & local model & $\mu(a)$ & $\mu(b)$ & $\mu(c)$ & $\mu(d)$ \\
\hline $\mathrm{A}_{p-1}$ & {$\left[\mathrm{I}_{n-p-0}\right]$} & $\left(t^{n}+x^{2}\right)\left(t^{p}+(x-\alpha)^{2}\right)(x-1)$ & 0 & 0 & $n+p$ & $n+p$ \\
\hline $\mathrm{D}_{p+4}$ & {$\left[\mathrm{I}_{n}-\mathrm{I}_{p}^{*}\right]$} & $\left(t^{n}+(x-1)^{2}\right)\left(t^{p+2}+x^{2}\right)(x+t)$ & 2 & 3 & $6+n+p$ & $6+n+p$ \\
\hline $\mathrm{E}_{6}$ & {$\left[\mathrm{IV}^{*}-\mathrm{I}_{n}\right]$} & $\left(t^{4}+x^{3}\right)\left(t^{n}+(x-1)^{2}\right)$ & $4+n$ & 4 & $8+n$ & $8+n$ \\
\hline $\mathrm{E}_{7}$ & {$\left[\mathrm{III}^{*}-\mathrm{I}_{n}\right]$} & $x\left(t^{3}+x^{2}\right)\left(t^{n}+(x-1)^{2}\right)$ & 3 & $6+n$ & $9+n$ & $9+n$ \\
\hline $\mathrm{E}_{8}$ & {$\left[\mathrm{II}^{*}-\mathrm{I}_{n}\right]$} & $\left(t^{5}+x^{3}\right)\left(t^{n}+(x-1)^{2}\right)$ & $5+n$ & 5 & $10+n$ & $10+n$ \\
\hline
\end{tabular}

Table 1: Genus-two models for ADE singularities.

As mentioned before, in general the resolution consists of a series of base blow-ups. Each curve is characterized by an integer equal to minus its self-intersection number, ${ }^{6}$ and by the gauge algebra factor it supports. This algebra is identified after checking for the presence of monodromies following the formalism of [28]. In order to determine the matter content it is also important to give the intersection pattern of the blow-ups. Applying the resolution procedure, we obtain all these data. The results match those obtained in [2]. Below we present two typical examples where we consider both the $E_{8} \times E_{8}$ and the $\operatorname{Spin}(32) / \mathbb{Z}_{2}$ heterotic string.

\section{$\left[\mathrm{II}^{*}-\mathrm{I}_{\mathrm{n}}\right]$ model and $\mathrm{E}_{8}$ singularity}

The pattern of curves and self-intersection numbers is efficiently determined using the toric geometry techniques reviewed in the preceding section. For the $E_{8} \times E_{8}$ heterotic we find:

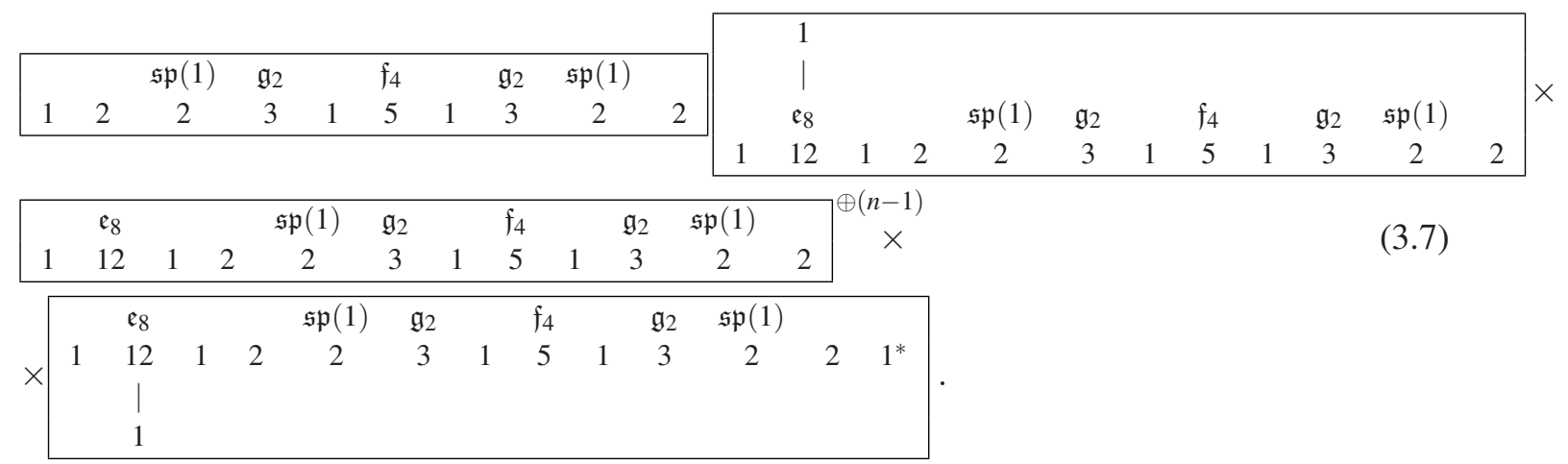

This result agrees with the theory of $k=10+n$, with $n \geq 1$, pointlike instantons on the $\mathrm{E}_{8}$ singularity as given in [2]. Deleting the node associated to $t=0$, gives the tensor branch description of a 6d SCFT embedded in the LST. This is the situation which was implicitly assumed in [22].

\footnotetext{
${ }^{6}$ After the resolutions, the curve $t=0$ has self-intersection -1 . We label this curve by $1^{*}$, instead of 1 , to indicate that it existed already before the base blow-ups.
} 
Starting from the $\operatorname{Spin}(32) / \mathbb{Z}_{2}$ heterotic string the pattern of gauge factors and self-intersection numbers turns out to be:

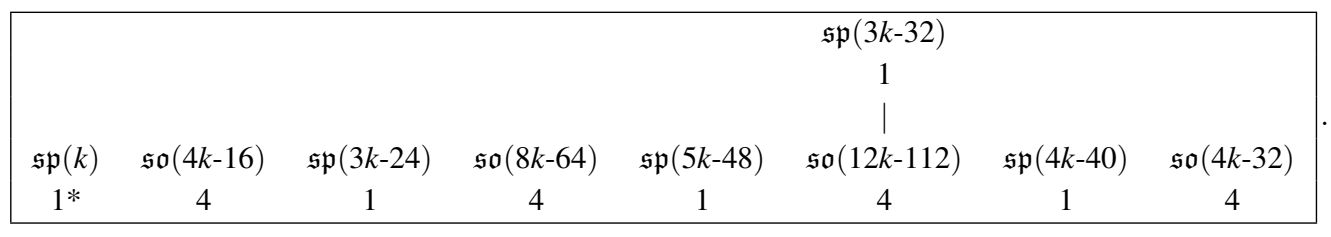

The first factor $\mathfrak{s p}(k)$ arises from the singularity at $t=0$ which, before the base blow-ups, is nonminimal only at $u=t=0$. The total number of base blow-ups is eight. Notice that the structure of the intersections conforms to the extended Dynkin diagram of $E_{8}$, in agreement with the analysis in [29]. Dropping the node corresponding to $t=0$, gives the tensor branch of a 6d SCFT embedded in the LST, with the $\mathfrak{s p}(k)$ remaining as a flavor symmetry.

$\left[\mathrm{I}_{0}^{*}-\mathrm{I}_{n}\right]$ model and $\mathrm{D}_{4}$ singularity

In the $E_{8} \times E_{8}$ case the resolution gives

\begin{tabular}{|c|c|c|c|c|c|c|c|c|c|c|c|}
\hline & & $\mathfrak{s p}(1)$ & $\mathfrak{g}_{2}$ & & $\mathfrak{s o}(8)$ & & $h^{\oplus}(n-$ & $\overline{\mathfrak{g}_{2}}$ & $\mathfrak{s p}(1)$ & & \\
\hline 1 & 2 & 2 & 3 & 1 & 4 & 1 & & 3 & 2 & 2 & $1^{*}$ \\
\hline
\end{tabular}

This result agrees with the theory of $k=6+n, n \geq 1$, pointlike instantons on the $\mathrm{D}_{4}$ singularity obtained in [2]. When $n=0$ we instead find

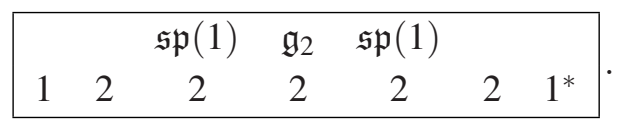

For the $\operatorname{Spin}(32) / \mathbb{Z}_{2}$ heterotic string, the resolution leads to

\begin{tabular}{|c|c|}
\hline \multicolumn{2}{|c|}{$n=0$} \\
\hline $\mathfrak{s p}(6)$ & $\mathfrak{s o}(7)$ \\
\hline $1^{*}$ & 1 \\
\hline
\end{tabular}
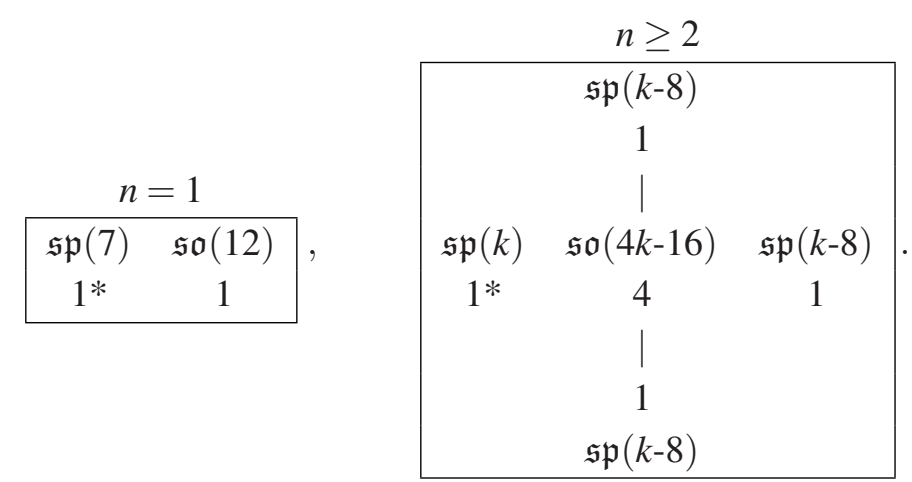

The number of blow-ups is one for $n=0,1$ and four for $n \geq 2$.

\subsection{Non-geometric models and duality web}

As seen in the previous section, in models corresponding to small instantons on ADE singularities, the explicit formulation of heterotic/F-theory duality in terms of the map between genus-two and $\mathrm{K} 3$ fibrations confirms the results expected from the monodromies of the moduli fields. We now turn to heterotic models with monodromies which are non-geometric in all T-duality frames. This is the most interesting situation, since a priori it is not clear if such degenerations are allowed. 


\begin{tabular}{|c|c|}
\hline$\mu(c)$ & dual models \\
\hline \hline 4 & {$\left[\mathrm{I}_{0}-\mathrm{IV}\right],[\mathrm{II}-\mathrm{II}]$} \\
\hline 5 & {$\left[\mathrm{IV}-\mathrm{I}_{1}\right],[\mathrm{II}-\mathrm{III}]$} \\
\hline 6 & {$\left[\mathrm{I}_{0}-\mathrm{I}_{0}^{*}\right],[\mathrm{III}-\mathrm{III}],[\mathrm{IV}-\mathrm{II}]$} \\
\hline 7 & {$\left[\mathrm{I}_{0}^{*}-\mathrm{I}_{1}\right],[\mathrm{IV}-\mathrm{III}]$} \\
\hline 8 & {$\left[\mathrm{I}_{0}-\mathrm{IV}^{*}\right],\left[\mathrm{I}_{0}^{*}-\mathrm{II}\right]$} \\
\hline 9 & {$\left[\mathrm{I}_{0}-\mathrm{III}^{*}\right],\left[\mathrm{I}_{0}^{*}-\mathrm{III}\right]$} \\
\hline 10 & {$\left[\mathrm{I}_{0}-\mathrm{II}^{*}\right],\left[\mathrm{I}_{0}^{*}-\mathrm{IV}\right]$} \\
\hline 11 & {$\left[\mathrm{II}_{-}-\mathrm{III}^{*}\right],\left[\mathrm{IV}{ }^{*}-\mathrm{III}\right]$} \\
\hline
\end{tabular}

Table 2: Dual models: the NU degenerations in the same row give rise to the same theories after resolution of the dual F-theory model.

A simple example of a non-geometric degeneration is the Namikawa-Ueno [III - III] singularity which has monodromy:

$$
\tau \rightarrow \frac{\rho}{\beta^{2}-\rho \tau}, \quad \rho \rightarrow \frac{\tau}{\beta^{2}-\rho \tau}, \quad \beta \rightarrow-\frac{\beta}{\beta^{2}-\rho \tau}
$$

When $\beta=0$ we obtain a "double elliptic" fibration which encircling the heterotic degeneration produces the monodromy $\tau \rightarrow-1 / \tau, \rho \rightarrow-1 / \rho$. The equation for the hyperelliptic curve for the [III - III] singularity is:

$$
y^{2}=x(x-1)\left(x^{2}+t\right)\left[(x-1)^{2}+t\right] .
$$

Applying the resolution procedure gives the same six-dimensional theory as $\left[\mathrm{I}_{0}-\mathrm{I}_{0}^{*}\right]$, c.f. (3.10) and (3.11), namely the theory of six small instantons on a $\mathrm{D}_{4}$ singularity. We have found that, in several non-geometric models of type 2 in the NU list, the dual CY admits a smooth resolution and, moreover, the resulting low energy physics is described by the theory of small instantons on ADE singularities.

As explained in [22], models with the same resolution such as [III $-\mathrm{III}]$ and $\left[\mathrm{I}_{0}-\mathrm{I}_{0}^{*}\right]$, can be related by certain duality moves. As a rule, such dual models appear when the sum of the vanishing orders of the discriminant for their two Kodaira components, or equivalently the vanishing order $\mu(c)$, is the same. In table 2 we display all the models satisfying this condition and admitting dual smooth Calabi-Yau resolutions. For all the models in table 2 we explicitly performed the F-theory resolution. For both heterotic strings, we verified that for all the degenerations in a row the same theory arises. The $\left[\mathrm{IV}^{*}-\mathrm{II}\right]$ model was originally included among the duals at $\mu(c)=10$. However, in the $\operatorname{Spin}(32) / \mathbb{Z}_{2}$ heterotic string its resolution differs from that of $\left[\mathrm{I}_{0}-\mathrm{II}^{*}\right]$ and closer inspection shows that this is also the case in the $E_{8} \times E_{8}$ heterotic string. Nonetheless, the theories could be connected by RG flow [4, 30]. A similar situation arises for the [IV - IV] model at $\mu(c)=8$ [9]. 


\section{A catalog of T-fects}

In this section, we summarize our findings for the Namikawa-Ueno models for which we could construct the dual CY resolution. Altogether there is a total of 49 models out of the 120 entries in the NU classification. The resulting patterns in the $E_{8} \times E_{8}$ case were thoroughly reported in [22] and for the $\operatorname{Spin}(32) / \mathbb{Z}_{2}$ heterotic string they appeared in [9]. Here we only present a few examples in both heterotic string theories.

\subsection{Elliptic type 1}

The elliptic type $1 \mathrm{NU}$ degenerations are characterized by a monodromy action that mixes the three moduli. Even though the corresponding heterotic models lack a geometric interpretation, the dual F-theory resolutions are similar to those discussed in the previous section. In table 3 we gather the models whose F-theory duals admit a smooth CY resolution. For example, the resolution for the [IX -1$]$ degeneration in the $E_{8} \times E_{8}$ heterotic is given by

\begin{tabular}{|c|c|c|c|c|c|c|c|c|c|c|c|c|c|c|c|c|}
\hline & $\mathfrak{s u}(2)$ & $\mathfrak{s o}(7)$ & $\mathfrak{s u}(2)$ & & $\mathfrak{e}_{7}$ & & & $\mathfrak{s p}(1)$ & $\mathfrak{g}_{2}$ & & $\mathfrak{f}_{4}$ & & $\mathfrak{g}_{2}$ & $\mathfrak{s p}(1)$ & & \\
\hline 1 & 2 & 3 & 2 & 1 & 8 & 1 & 2 & 2 & 3 & 1 & 5 & 1 & 3 & 2 & 2 & $1 *$ \\
\hline
\end{tabular}

In the $\operatorname{Spin}(32) / \mathbb{Z}_{2}$ we find

\begin{tabular}{|ccccccc|}
\hline $\mathfrak{s p}(8)$ & $\mathfrak{s o}(20)$ & $\mathfrak{s p}(4)$ & $\mathfrak{s o}(12)$ & & $\mathfrak{s u}(2)$ & $\mathfrak{s o}(7)$ \\
$1 *$ & 4 & 1 & 4 & 1 & 2 & 3 \\
\hline
\end{tabular}

\begin{tabular}{|c|c|c|c|c|}
\hline NU model & $\mu(a)$ & $\mu(b)$ & $\mu(c)$ & $\mu(d)$ \\
\hline \hline$\left[\mathrm{I}_{0-0-0}\right]$ & 0 & 0 & 0 & 0 \\
\hline$[\mathrm{V}]$ & 2 & 3 & 5 & 6 \\
\hline$[\mathrm{VII}]$ & 2 & 3 & 5 & 6 \\
\hline$[\mathrm{VIII}-1]$ & $\infty$ & $\infty$ & 4 & $\infty$ \\
\hline$[\mathrm{IX}-1]$ & $\infty$ & $\infty$ & 8 & $\infty$ \\
\hline
\end{tabular}

Table 3: Elliptic type 1 models.

\subsection{Elliptic type 2}

Type 2 models in the NU list comprise degenerations of type $\left[\mathrm{K}_{1}-\mathrm{K}_{2}-m\right]$, with $m \geq 0$, where $\mathrm{K}_{1}$ and $\mathrm{K}_{2}$ are one of the Kodaira type singularities for the two genus-one components of the genus-two surface $\Sigma$, plus additional sporadic models denoted as $[2 \mathrm{~K}-m]$ and $\left[\mathrm{K}_{1}-\mathrm{K}_{2}-\alpha\right]$. None of the latter, nor any of the models with $m \neq 0$, lead to a dual CY admitting a smooth crepant resolution. We find 20 models that can be resolved. They are displayed in table 4 using again the notation $\left[\mathrm{K}_{1}-\mathrm{K}_{2}-0\right] \equiv\left[\mathrm{K}_{1}-\mathrm{K}_{2}\right]$. The models of type $\left[\mathrm{I}_{0}-\mathrm{K}_{2}\right]$ correspond to a configuration 


\begin{tabular}{|c|c|c|c|c||c|c|c|c|c|}
\hline NU model & $\mu(a)$ & $\mu(b)$ & $\mu(c)$ & $\mu(d)$ & NU model & $\mu(a)$ & $\mu(b)$ & $\mu(c)$ & $\mu(d)$ \\
\hline \hline$\left[\mathrm{I}_{0}-\mathrm{I}_{0}\right]$ & 0 & 0 & 0 & 0 & {$[\mathrm{II}-\mathrm{IV}]$} & 3 & 3 & 6 & 6 \\
\hline$\left[\mathrm{I}_{0}-\mathrm{II}\right]$ & 1 & 1 & 2 & 2 & {$\left[\mathrm{I}_{0}^{*}-\mathrm{II}\right]$} & 3 & 4 & 8 & 8 \\
\hline$\left[\mathrm{I}_{0}-\mathrm{III}\right]$ & 1 & 2 & 3 & 3 & {$\left[\mathrm{II}-\mathrm{IV}{ }^{*}\right]$} & 5 & 5 & 10 & 10 \\
\hline$\left[\mathrm{I}_{0}-\mathrm{IV}\right]$ & 2 & 2 & 4 & 4 & {$\left[\mathrm{II}-\mathrm{III}{ }^{*}\right]$} & 4 & 7 & 11 & 11 \\
\hline$\left[\mathrm{I}_{0}-\mathrm{I}_{0}^{*}\right]$ & 2 & 3 & 6 & 6 & {$[\mathrm{III}-\mathrm{III}]$} & 2 & 4 & 6 & 6 \\
\hline$\left[\mathrm{I}_{0}-\mathrm{IV}^{*}\right]$ & 4 & 4 & 8 & 8 & {$[\mathrm{IV}-\mathrm{III}]$} & 3 & 4 & 7 & 7 \\
\hline$\left[\mathrm{I}_{0}-\mathrm{III}{ }^{*}\right]$ & 3 & 6 & 9 & 9 & {$\left[\mathrm{I}_{0}^{*}-\mathrm{III}\right]$} & 3 & 5 & 9 & 9 \\
\hline$\left[\mathrm{I}_{0}-\mathrm{II}{ }^{*}\right]$ & 5 & 5 & 10 & 10 & {$[\mathrm{IV} *-\mathrm{III}]$} & 5 & 6 & 11 & 11 \\
\hline$[\mathrm{II}-\mathrm{II}]$ & 2 & 2 & 4 & 4 & {$[\mathrm{IV}-\mathrm{IV}]$} & 4 & 4 & 8 & 8 \\
\hline$[\mathrm{II}-\mathrm{III}]$ & 2 & 3 & 5 & 5 & {$\left[\mathrm{I}_{0}^{*}-\mathrm{IV}\right]$} & 4 & 5 & 10 & 10 \\
\hline
\end{tabular}

Table 4: Elliptic type 2 models.

of $k=\mu(c)$ pointlike instantons on the $\mathrm{K}_{2}$ singularity. The remaining models are non-geometric since their monodromies involve non-trivial actions on the torus volume. However, as discussed in section 3.3, many of these models lead to the same resolutions as the geometric ones.

\subsection{Parabolic type 3}

In this class there are additional models for which the monodromy factorizes into the product of two monodromies — one Kodaira type for each handle of $\Sigma$ —one of which is either $\mathrm{I}_{n}$ or $\mathrm{I}_{n}^{*}$ (the only parabolic elements in the Kodaira list) and the other is of elliptic type. There are also models labeled $\left[\mathrm{K}_{1}-\mathrm{II}_{n}\right]$ or $\left[\mathrm{K}_{1}-\mathrm{II}_{n}^{*}\right]$ that mix all moduli but have a Kodaira type $\mathrm{K}_{1}$ monodromy for $\tau$.

The 19 models that can be resolved are listed in table 5. They admit a resolution for all $n$. The models of type $\left[\mathrm{I}_{n}-\mathrm{K}_{2}\right]$ or $\left[\mathrm{K}_{1}-\mathrm{I}_{n}\right]$ again correspond to $k=\mu(d)$ pointlike instantons on the $\mathrm{K}_{i}$ singularity. In this class we also discover dual models. Concretely, starting with the fifth row in table 5, the models in the same row have the same resolution. We illustrate the resolutions in this class with the $\left[\mathrm{IV}^{*}-\mathrm{II}_{n}\right]$ model. In the $E_{8} \times E_{8}$ heterotic string we obtain

\begin{tabular}{|c|c|c|c|c|c|c|c|c|c|c|c|c|c|}
\hline & $\mathfrak{s u}(2)$ & $\mathfrak{s o}(7)$ & $\mathfrak{s u}(2)$ & & $\mathfrak{e}_{6}$ & & $\mathfrak{w} \mathfrak{u}(3)$ & $\overline{\mathfrak{f}_{4}}$ & & $\mathfrak{g}_{2}$ & $\overline{\mathfrak{s p}}(1)$ & & \\
\hline 1 & 2 & 3 & 2 & 1 & 6 & 1 & 1 & 5 & 1 & 3 & 2 & 2 & $1 *$ \\
\hline
\end{tabular}

In the $\operatorname{Spin}(32) / \mathbb{Z}_{2}$ heterotic string we deduce

\begin{tabular}{|ccccc|}
\hline $\mathfrak{s p}(n+7)$ & $\mathfrak{s o}(4 n+16)$ & $\mathfrak{s p}(3 n+1)$ & $\mathfrak{s u}(4 n+2)$ & $\mathfrak{s u}(2 n+2)$ \\
$1 *$ & 4 & 1 & 2 & 2 \\
\hline
\end{tabular}




\begin{tabular}{|c|c|c|c|c||c|c|c|c|c|}
\hline NU model & $\mu(a)$ & $\mu(b)$ & $\mu(c)$ & $\mu(d)$ & NU model & $\mu(a)$ & $\mu(b)$ & $\mu(c)$ & $\mu(d)$ \\
\hline \hline$\left[\mathrm{I}_{n-0}-0\right]$ & 0 & 0 & $n$ & $n$ & {$\left[\mathrm{II}-\mathrm{I}_{n}\right]$} & $1+n$ & 1 & $2+n$ & $2+n$ \\
\hline$\left[\mathrm{III}-\mathrm{I}_{n}\right]$ & 1 & $2+n$ & $3+n$ & $3+n$ & {$\left[\mathrm{III}-\mathrm{II}_{n}\right]$} & 1 & $2+n$ & $3+n$ & $4+n$ \\
\hline$\left[\mathrm{IV}-\mathrm{I}_{n}\right]$ & $2+n$ & 2 & $4+n$ & $4+n$ & {$\left[\mathrm{IV}-\mathrm{II}_{n}\right]$} & $2+n$ & 2 & $4+n$ & $5+n$ \\
\hline$\left[\mathrm{II}_{n-0}\right]$ & 2 & 3 & $5+n$ & $6+n$ & & & & & \\
\hline$\left[\mathrm{I}_{n}-\mathrm{I}_{0}^{*}\right]$ & 2 & 3 & $6+n$ & $6+n$ & {$\left[\mathrm{I}_{0}-\mathrm{I}_{n}^{*}\right]$} & 2 & 3 & $6+n$ & $6+n$ \\
\hline$\left[\mathrm{IV}^{*}-\mathrm{I}_{n}\right]$ & $4+n$ & 4 & $8+n$ & $8+n$ & {$\left[\mathrm{II}-\mathrm{I}_{n}^{*}\right]$} & 3 & 4 & $8+n$ & $8+n$ \\
\hline$\left[\mathrm{III}^{*}-\mathrm{I}_{n}\right]$ & 3 & $6+n$ & $9+n$ & $9+n$ & {$\left[\mathrm{III}-\mathrm{I}_{n}^{*}\right]$} & 3 & 5 & $9+n$ & $9+n$ \\
\hline$\left[\mathrm{II}^{*}-\mathrm{I}_{n}\right]$ & $5+n$ & 5 & $10+n$ & $10+n$ & {$\left[\mathrm{IV}-\mathrm{I}_{n}^{*}\right]$} & 4 & 5 & $10+n$ & $10+n$ \\
\hline$\left[\mathrm{IV}^{*}-\mathrm{II}_{n}\right]$ & $3+n$ & 4 & $7+n$ & $9+n$ & {$\left[\mathrm{II}-\mathrm{II}_{n}^{*}\right]$} & $3+n$ & 4 & $7+n$ & $9+3 n$ \\
\hline$\left[\mathrm{III}^{*}-\mathrm{II}_{n}\right]$ & 3 & $5+n$ & $8+n$ & $11+n$ & {$\left[\mathrm{III}-\mathrm{II}_{n}^{*}\right]$} & 3 & $5+n$ & $8+n$ & $10+2 n$ \\
\hline
\end{tabular}

Table 5: Parabolic type 3 models.

\subsection{Parabolic type 4}

This class includes degenerations associated to parabolic Kodaira singularities for both the genus-one components of $\Sigma$, of type $\left[\mathrm{K}_{1}-\mathrm{K}_{2}-m\right]$ with $\mathrm{K}_{1,2}=\mathrm{I}_{n}, \mathrm{I}_{n}^{*}$, plus additional degenerations of type $\left[2 \mathrm{~K}_{1}-m\right],\left[\mathrm{II}_{n-p}\right]$, and $\left[\mathrm{III}_{n}\right]$. Only the three models shown in table 6 admit a dual smooth resolution. For instance, the resolution of $\left[\mathrm{II}_{n-3}\right], n>3$, singularity for the $E_{8} \times E_{8}$ heterotic string reads

\begin{tabular}{|c|c|c|c|c|c|c|c|c|c|c|}
\hline & $\mathfrak{s u}(2)$ & $\mathfrak{s o}(7)$ & $\mathfrak{s o}(9$ & ) $\mathfrak{s p}(1)$ & $\mathfrak{s o}(11)$ & $\mathfrak{s p}(2)$ & $\mathfrak{s o}(13)$ & $\mathfrak{s p}(3)$ & $\mathfrak{s o}(14)$ & $\mathfrak{s p ( 3 )}$ \\
\hline 1 & 2 & 3 & 1 & 1 & 4 & 1 & 4 & 1 & 4 & 1 \\
\hline & $\mathfrak{s o}(13$ & $\mathfrak{s p}(2)$ & $\mathfrak{s o}(11)$ & $\mathfrak{s p}(1)$ & $\mathfrak{s o ( 9 )}$ & $\mathfrak{g}_{2}$ & $\mathfrak{p}(1)$ & & & \\
\hline & 4 & 1 & 4 & 1 & 4 & 3 & 2 & $1 *$ & & \\
\hline
\end{tabular}

This result is similar to the resolution of $k>12$ instantons on a $D_{7}$ singularity [3]. Such similarity is also observed in the $\operatorname{Spin}(32) / \mathbb{Z}_{2}$ heterotic string. For example, in the case of the same $\left[\mathrm{II}_{n-3}\right]$, $n>3$, degeneration we obtain

\begin{tabular}{|cccccc|}
\hline & $\mathfrak{s p}(n+2)$ & & & & \\
& 1 & & & & \\
& $\mid$ & & & & \\
$\mathfrak{s p}(n+8)$ & $\mathfrak{s o}(4 n+20)$ & $\mathfrak{s p}(2 n+2)$ & $\mathfrak{s o}(4 n+4)$ & $\mathfrak{s p}(2 n-6)$ & $\mathfrak{s u}(2 n-6)$ \\
$1^{*}$ & 4 & 1 & 4 & 1 & 2 \\
\hline
\end{tabular}

\subsection{Parabolic type 5}

The final class in the NU list is that of parabolic type 5 models, which includes just 6 degenerations. Only the two of them collected in table 7 admit a smooth resolution presented in the 


\begin{tabular}{|c|c|c|c|c|}
\hline NU model & $\mu(a)$ & $\mu(b)$ & $\mu(c)$ & $\mu(d)$ \\
\hline \hline$\left[\mathrm{I}_{n-p-0}\right]$ & 0 & 0 & $n+p$ & $n+p$ \\
\hline$\left[\mathrm{I}_{n}-\mathrm{I}_{p}^{*}\right]$ & 2 & 3 & $6+n+p$ & $6+n+p$ \\
\hline$\left[\mathrm{II}_{n-p}\right]$ & 2 & 3 & $5+n+p$ & $6+n+p$ \\
\hline
\end{tabular}

Table 6: Parabolic type 4 models.

following. The parabolic type $5\left[\mathrm{II}_{n-p}\right]$ is not the same as the one listed in table 6 . Their sextics are distinct and lead to different resolutions. For the $E_{8} \times E_{8}$ heterotic string, the resolution of the type $5\left[\mathrm{II}_{n-3}\right], n>3$, yields

\begin{tabular}{|c|c|c|c|c|c|c|c|c|c|c|c|c|}
\hline & $\mathfrak{s u}(2)$ & $\mathfrak{s o}(7)$ & $\mathfrak{s u}(2)$ & $\mathfrak{s o}(12)$ & $\mathfrak{s p}(3)$ & $\mathfrak{s o}(14)$ & $\mathfrak{s p}(3)$ & $\mathfrak{s o}(14)$ & $\mathfrak{s p}(3)$ & $\mathfrak{s o}(14)$ & $\mathfrak{s p}(3)$ & $(n-$ \\
\hline 1 & 2 & 3 & 1 & 4 & 1 & 4 & 1 & 4 & 1 & 4 & 1 & X \\
\hline$x$ & $\mathfrak{s o}(13)$ & $\mathfrak{s p}(2)$ & $\mathfrak{s o}(11)$ & $\mathfrak{s p}(1)$ & $\mathfrak{s o}(9)$ & $\mathfrak{g}_{2}$ & $\mathfrak{s p}(1)$ & & & & & \\
\hline$x$ & 4 & 1 & 4 & 1 & 4 & 3 & 2 & $1 *$ & & & & \\
\hline
\end{tabular}

whereas the resolution of the same model in the $\operatorname{Spin}(32) / \mathbb{Z}_{2}$ heterotic string gives

\begin{tabular}{|cccccc|}
\hline & $\mathfrak{s p}(n+1)$ & & & & \\
& 1 & & & & \\
& $\mid$ & & & & \\
$\mathfrak{s p}(n+8)$ & $\mathfrak{s o}(4 n+20)$ & $\mathfrak{s p}(2 n+3)$ & $\mathfrak{s o}(4 n+8)$ & $\mathfrak{s p}(2 n-3)$ & $\mathfrak{s u}(2 n-2)$ \\
$1 *$ & 4 & 1 & 4 & 1 & 2 \\
\hline
\end{tabular}

The above results are evidently different from the resolutions of the type $4\left[\mathrm{II}_{n-3}\right]$ displayed in (4.5) and (4.6).

\begin{tabular}{|c|c|c|c|c|}
\hline NU model & $\mu(a)$ & $\mu(b)$ & $\mu(c)$ & $\mu(d)$ \\
\hline \hline$\left[\mathrm{I}_{n-p-q}\right]$ & 0 & 0 & $n+p+q$ & $n+p+q$ \\
\hline$\left[\mathrm{II}_{n-p}\right] p=2 k+l, l=0,1$ & 2 & 3 & $5+l+2 k+n$ & $6+l+2 k+n$ \\
\hline
\end{tabular}

Table 7: Parabolic type 5 models.

\section{Final comments}

In this work, we have studied heterotic compactifications with six dimensional T-fects leaving an $E_{8} \times E_{7}$ or a $\operatorname{Spin}(28) \times S U(2) / \mathbb{Z}_{2}$ subgroup unbroken. We have focused on configurations which are locally described by a $T^{2}$ fibration over a complex one-dimensional base with a smooth, up to the degeneration points, $S U(2)$ structure bundle, patched together using arbitrary elements of $S O^{+}(2,3, \mathbb{Z})$-an order four subgroup of the T-duality group $O(2,3, \mathbb{Z})$. Generically, this gives rise 
to backgrounds without a global classical geometric interpretation. At certain points in the base, the fibration, or bundle data on it, will degenerate and will no longer have-in any T-duality frame-an interpretation in terms of the heterotic string on a smooth $T^{2}$ with a smooth vector bundle on top. Our goal in this paper has been to characterize the physics arising from such singular points.

We have exploited the fact that for backgrounds preserving $E_{8} \times E_{7}$ or $\operatorname{Spin}(28) \times S U(2) / \mathbb{Z}_{2}$, the geometric data of the heterotic string on $T^{2}$ can be encoded in the geometry of a genus-two (sextic) Riemann surface. One can then define vacua by fibering this genus-two Riemann surface over a complex one-dimensional base. For monodromies in $S O^{+}(2,3, \mathbb{Z})$, or equivalently $\operatorname{Sp}(4, \mathbb{Z})$, one can classify the ways in which such fibration can degenerate $[15,16]$. Using heterotic/F-theory duality to reinterpret these degenerations of the sextic as degenerations of dual F-theory K3s fibered over the same base, we are able to read off the low-energy physics at the degeneration point.

We performed a systematic analysis on the full set of sextic degenerations. Remarkably, we found that many (non-)geometric degenerations are described by the same low-energy physics. Often these are given by the long-understood configurations of pointlike instantons sitting on ADE singularities. It would be very interesting to understand the origin of this phenomenon in heterotic language without going to F-theory.

A second notable finding is that not all of the possible sextic degenerations admit an F-theory dual that can be smoothed out in a crepant way by a finite number of blow-ups. As explained in section 3, this follows from the fact that in these cases the F-theory configuration is associated with non-minimal Weierstraß models in complex codimension one (after we performed already some base blow-ups). In these cases, we cannot determine the low-energy physics using F-theory techniques, since the dynamics of F-theory on such backgrounds is unknown. Assuming that these vacua are consistent too, it would be very important to find out which kind of theories arise from these backgrounds in the IR. They may give rise to free or trivial theories, or alternatively to interacting SCFTs without a tensor branch - at least no geometrically manifest tensor branch. Understanding these 'non-minimal models' is an open problem that deserves further attention.

\section{References}

[1] C. Vafa, "Evidence for F theory," Nucl.Phys. B469 (1996) 403-418, hep-th / 9602022.

[2] P. S. Aspinwall and D. R. Morrison, "Point - like instantons on K3 orbifolds," Nucl. Phys. B503 (1997) 533-564, hep-th/9705104.

[3] K. A. Intriligator, "New string theories in six-dimensions via branes at orbifold singularities," $A d v$. Theor. Math. Phys. 1 (1998) 271-282, hep-th/9708117.

[4] J. J. Heckman, D. R. Morrison, and C. Vafa, "On the Classification of 6D SCFTs and Generalized ADE Orbifolds," JHEP 05 (2014) 028, 1312 . 574 6. [Erratum: JHEP06,017(2015)].

[5] J. J. Heckman, D. R. Morrison, T. Rudelius, and C. Vafa, "Atomic Classification of 6D SCFTs," Fortsch. Phys. 63 (2015) 468-530, 1502.05405.

[6] M. Del Zotto, J. J. Heckman, A. Tomasiello, and C. Vafa, “6d Conformal Matter," JHEP 02 (2015) $054,1407.6359$.

[7] L. Bhardwaj, "Classification of 6d $\mathscr{N}=(1,0)$ gauge theories," JHEP 11 (2015) 002, 1502.06594. 
[8] L. Bhardwaj, M. Del Zotto, J. J. Heckman, D. R. Morrison, T. Rudelius, and C. Vafa, "F-theory and the Classification of Little Strings," Phys. Rev. D93 (2016), no. 8 086002, 1511.05565.

[9] A. Font and C. Mayrhofer, "Non-Geometric Vacua of the $\operatorname{Spin}(\mathbf{3 2}) / \mathbb{Z}_{\mathbf{2}}$ Heterotic String and Little String Theories," 1708.05428.

[10] N. Seiberg, "New theories in six-dimensions and matrix description of M theory on $\mathrm{T} * * 5$ and $\mathrm{T} * * 5 /$ Z(2)," Phys. Lett. B408 (1997) 98-104, hep-th/9705221.

[11] D. R. Morrison and C. Vafa, "Compactifications of F theory on Calabi-Yau threefolds. 1," Nucl.Phys. B473 (1996) 74-92, hep-th/ 9602114.

[12] A. Malmendier and D. R. Morrison, "K3 surfaces, modular forms, and non-geometric heterotic compactifications,” Lett. Math. Phys. 105 (2015), no. 8 1085-1118, 1406.4873.

[13] J. Gu and H. Jockers, "Nongeometric F-theory-heterotic duality," Phys.Rev. D91 (2015) 086007, 1412.5739.

[14] K. S. Narain, "New Heterotic String Theories in Uncompactified Dimensions < 10," Phys. Lett. B169 (1986) 41-46.

[15] A. P. Ogg, “On pencils of curves of genus two,” Topology (1966) 355-362.

[16] Y. Namikawa and K. Ueno, "The complete classification of fibres in pencils of curves of genus two," Manuscripta Math. 9 (1973), no. 2 143-186.

[17] P. Berglund and P. Mayr, "Heterotic string / F theory duality from mirror symmetry," Adv. Theor. Math. Phys. 2 (1999) 1307-1372, hep-th/9811217.

[18] R. Friedman, J. Morgan, and E. Witten, "Vector bundles and F theory," Commun.Math.Phys. 187 (1997) 679-743, hep-th/9701162.

[19] A. Clingher and C. F. Doran, "Lattice Polarized K3 Surfaces and Siegel Modular Forms," Adv. Math. 231 (2012) 172, arXiv:1004.3503 [math.AG] .

[20] P. S. Aspinwall, “K3 Surfaces and String Duality,” 9611137.

[21] J. H. Bruinier, G. van der Geer, G. Harder, and D. Zagier, The 1-2-3 of Modular Forms. Springer Berlin Heidelberg, 2008.

[22] A. Font, I. García-Etxebarria, D. Lust, S. Massai, and C. Mayrhofer, "Heterotic T-fects, 6D SCFTs, and F-Theory," JHEP 08 (2016) 175, 1603.09361.

[23] J.-I. Igusa, “On Siegel Modular Forms of Genus Two,” American Journal of Mathematics 84 (1962), no. 1 175-200.

[24] J. McOrist, D. R. Morrison, and S. Sethi, "Geometries, Non-Geometries, and Fluxes," Adv.Theor.Math.Phys. 14 (2010) 1004.5447.

[25] I. García-Etxebarria, D. Lust, S. Massai, and C. Mayrhofer, "Ubiquity of non-geometry in heterotic compactifications," JHEP 03 (2017) 046, 1611 . 10291.

[26] K. Kodaira, “On compact analytic surfaces I-III,” Ann. of Math., 71 (1960), 111-152; 77 (1963), 563-626; 78 (1963), 1-40.

[27] W. Fulton, Introduction to toric varieties. No. 131. Princeton University Press, 1993.

[28] A. Grassi and D. R. Morrison, "Anomalies and the Euler characteristic of elliptic Calabi-Yau threefolds," Commun.Num.Theor.Phys. 6 (2012) 51-127, 1109.0042. 
[29] J. D. Blum and K. A. Intriligator, "New phases of string theory and 6-D RG fixed points via branes at orbifold singularities," Nucl. Phys. B506 (1997) 199-222, hep-th/9705044.

[30] J. J. Heckman, D. R. Morrison, T. Rudelius, and C. Vafa, “Geometry of 6D RG Flows,” JHEP 09 (2015) 052, 1505.00009 . 